\title{
A Panoramic Survey of Natural Language Processing in the Arab World
}

\author{
Kareem Darwish, Nizar Habash, \\ Mourad Abbas, Hend Al-Khalifa, Huseein T. Al-Natsheh, \\ Samhaa R. El-Beltagy, Houda Bouamor, Karim Bouzoubaa, \\ Violetta Cavalli-Sforza, Wassim El-Hajj, \\ Mustafa Jarrar, and Hamdy Mubarak
}

October 2020

\section{Natural Language and its Processing}

The term natural language refers to any system of symbolic communication (spoken, signed or written) that has evolved naturally in humans without intentional human planning and design. This distinguishes natural languages such as Arabic and Japanese from artificially constructed languages such as Esperanto or Python. Natural language processing (NLP), also called computational linguistics or human language technologies, is the sub-field of artificial intelligence (AI) focused on modeling natural languages to build applications such as speech recognition and synthesis, machine translation, optical character recognition (OCR), sentiment analysis (SA), question answering, dialogue systems, etc. NLP is a highly interdisciplinary field with connections to computer science, linguistics, cognitive science, psychology, mathematics and others.

Some of the earliest AI applications were in NLP (e.g., machine translation); and the last decade (2010-2020) in particular has witnessed an incredible increase in quality, matched with a rise in public awareness, use, and expectations of what may have seemed like science fiction in the past. NLP researchers pride themselves on developing language independent models and tools that can be applied to all human languages, e.g. machine translation systems can be built for a variety of languages using the same basic mechanisms and models. However, the reality is that some languages do get more attention (e.g., English and Chinese) than others (e.g., Hindi and Swahili). Arabic, the primary language of the Arab world and the religious language of millions of non-Arab Muslims is somewhere in the middle of this continuum. Though Arabic NLP has many challenges, it has seen many successes and developments.

Next we discuss Arabic's main challenges as a necessary background, and we present a brief history of Arabic NLP. We then survey a number of its research areas, and close with a critical discussion of the future of Arabic NLP. 


\section{Arabic and its Challenges}

Arabic today poses a number of modeling challenges for NLP: morphological richness, orthographic ambiguity, dialectal variations, orthographic noise, and resource poverty. We do not include issues of right-to-left Arabic typography, which is an effectively solved problem (although not universally implemented).

Morphological Richness Arabic words have numerous forms resulting from a rich inflectional system that includes features for gender, number, person, aspect, mood, case, and a number of attachable clitics. As a result, it is not uncommon to find single Arabic words that translate into five-word English sentences: وَسَيَدرُسُونَهَ wa+sa+ya-drus-uuna+ha 'and they will study it'. This challenge leads to a higher number of unique vocabulary types compared to English, which is challenging for machine learning models.

Orthographic Ambiguity The Arabic script uses optional diacritical marks to represent short vowels and other phonological information that is important to distinguish words from each other. These marks are almost never used outside of religious texts and children's literature, which leads to a high degree of ambiguity. Educated Arabs do not usually have a problem with reading undiacritized Arabic, but it is a challenge for Arabic learners and computers. This out-of-context ambiguity in Standard Arabic leads to a staggering 12 analyses per word on average: e.g. the readings of the word كتبت ktbt (no diacritics) includes كَتَنْتُ katabtu 'I wrote', كَتَبَتْ katabat 'she wrote', and the quite seman-

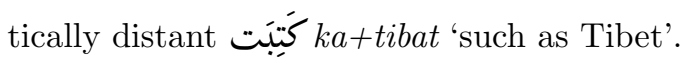

Dialectal Variation Arabic is also not a single language but rather a family of historically linked varieties, among which Standard Arabic is the official language of governance, education and the media, while the other varieties, socalled dialects, are the languages of daily use in spoken, and increasingly written, form. Arab children grow up learning their native dialects, such as Egyptian, Levantine, Gulf, or Moroccan Arabic, which have their own grammars and lexicons that differ from each other and from Standard Arabic. For example, the word for 'car' is سيارة sayyaara in Standard Arabic, عربية arabiyya in Egyptian

Arabic, مرهبة marhba in Tunisian Arabic, and in Gulf Arabic. The differences can be significant to the point that using Standard Arabic tools on dialectal Arabic leads to quite sub-optimal performance. For instance, [119] report that using a state-of-the-art tool for MSA morphological disambiguation on Gulf Arabic returns POS tag and lemma accuracy at about $72 \%$ and $64 \%$ respectively, compared to the performance on MSA, which is $96 \%$ for both [156].

Orthographic Inconsistency Standard and dialectal Arabic are both written with a high degree of spelling inconsistency, especially on social media: A 
third of all words in MSA comments online have spelling errors [201]; and dialectal Arabic has no official spelling standards, although there are efforts to develop such standards computationally, such as the work on CODA, or Conventional Orthography for Dialectal Arabic 95. Furthermore, Arabic can be encountered online written in other scripts, most notably, a romanization called Arabizi that attempts to capture the phonology of the words [57].

Resource Poverty Data is the bottleneck of NLP: this is true for rule-based approaches that need lexicons and carefully created rules; and for Machine Learning (ML) approaches that need corpora and annotated corpora. Although Arabic unannotated text corpora are quite plentiful, Arabic morphological analyzers and lexicons as well as annotated and parallel data in non-news genre and in dialects are limited.

None of the above-mentioned issues are unique to Arabic - e.g. Turkish and Finnish are morphologically rich; Hebrew is orthographically ambiguous; and many languages have dialectal variants. However, the combination and degree of these phenomena in Arabic creates a particularly challenging situation for NLP research and development. For more information on Arabic computational processing challenges, see [87, 102].

\section{A Brief History of NLP in the Arab World}

Historically, Arabic NLP can be said to have gone through three waves. The first wave was in the early 1980's with the introduction of Microsoft MS-DOS 3.3 with Arabic language support. In 1985 the first Arabic morphological analyzer was developed by Sakhr. Most of the research in that period focused on morphological analysis of Arabic text and using rule-based approaches. Sakhr has also continued leading research and development in Arabic computational linguistics by developing the first syntactic and semantic analyzer in 1992 and Arabic optical character recognition in 1995. Sakhr also produced many commercial products and solutions including Arabic to English machine translation, Arabic text-to-speech (TTS), and an Arabic search engine. This period almost exclusively focused on Standard Arabic with a few exceptions related to work on speech recognition [89.

The second wave was during the years 2000-2010. Arabic NLP gained increasing importance in the Western world especially after September 11. The USA funded large projects for companies and research centers to develop NLP tools for Arabic and its dialects including: machine translation, speech synthesis and recognition, information retrieval and extraction, text to speech, and named entity recognition [87, 102, 152. Most of the systems developed in that period used machine learning, which was on the rise in the field of NLP as a whole. In principle, ML required far less linguistic knowledge than rule-based approaches, and was fast and more accurate. However it needed a lot of data, some of which was not easy to collect, e.g. dialectal Arabic to English parallel texts. Arabic's rich morphology exacerbated the data dependence further. So, this period saw 
some successful instances of hybrid systems that combine rule-based morphological analyzers with ML disambiguation which relied on the then newly created Penn Arabic Treebank (PATB) 98, 128. The leading universities, companies and consortia at the time were: Columbia University, University of Maryland, IBM, BBN, SRI, the Linguistic Data Consortium (LDC), and European Language Resources Association (ELRA).

The third wave started in 2010, when the research focus on Arabic NLP came back to the Arab world. This period witnessed a proliferation of Arab researchers and graduate students interested in Arabic NLP and an increase in publications in top conferences from the Arab world. Active universities include New York University Abu Dhabi (NYUAD), American University in Beirut (AUB), Carnegie Mellon University in Qatar (CMUQ), King Saud University (KSU), Birzeit University (BZU), Cairo University, etc. Active research centers include Qatar Computing Research Institute (QCRI), King Abdulaziz City for Science and Technology (KACST), etc. It should be noted that there are many actively contributing researchers in smaller groups across the Arab world. This period also overlapped with two major independent developments: one is the rise of deep learning and neural models and the other is the rise of social media. The first development affected the direction of research pushing it further into the ML space; and the second led to the increase in social media data, which introduced many new challenge at a larger scale: more dialects and more noise. This period also witnessed a welcome increase in Arabic language resources and processing tools, and a heightened awareness of the importance of AI for the future of the region - e.g. the UAE now has a ministry for AI specifically. Finally, new young and ambitious companies such as Mawdoo3 are competing for a growing market and expectations in the Arab world. It's important to note that throughout the different waves, researchers of different backgrounds (Arab and non-Arab) contributed and continue to contribute to the developments in the Arabic NLP.

\section{Arabic Tools and Resources}

We organize this section on Arabic tools and resources into two parts: first, we discuss enabling technologies which are the basic resources and utilities that are not user-facing products; and, second, we discuss a number of advanced user-targeting applications.

\subsection{Arabic Enabling Resources and Technologies}

\subsubsection{Corpora and Lexical Resources}

Resource construction is a lengthy and costly task that requires significant teamwork among linguists, lexicographers, and publishers over an extended period of time. 
Corpora NLP relies heavily on the existence of corpora for developing and evaluating its models, and the performance of NLP applications directly depends on the quality of these corpora. Historically, Al-Khalil ibn Ahmad Al-Farahidi, was among the first to assemble a large collection of texts in the 8th century 197. Textual corpora are classified at a high level as annotated and unannotated corpora.

Annotated corpora are naturally a subset of unannotated corpora that have been enriched with additional information such as contextual morphological analyses, lemmas, diacritizations, part-of-speech tags, syntactic analyses, dialect IDs, named entities, sentiment, and even parallel translations. The more information, the costlier the process is to create such corpora. For Arabic, the main collections of annotated corpora were created in its second wave, mostly outside the Arab world. the most notable annotated resource is the LDC's Penn Arabic Treebank (PATB) [128, which provides a relatively large MSA corpus that is morphologically analyzed, segmented, lemmatized, tagged with fine-grained parts of speech, diacritized, and parsed. PATB has enabled much of the Arabic NLP research since its creation. The Prague Arabic Dependency Treebank (PADT) was the first dependency representation treebank for Arabic [188. The Columbia Arabic Treebank (CATiB) was an effort to develop a simplified dependency representation with a faster annotation scheme for MSA [99, 193]. The University of Leeds' Quranic Arabic Corpus is a beautifully constructed treebank that uses traditional morpho-syntactic analyses of the Holy Quran [72, 73]. With the rising interest in dialectal data, there have been many efforts to collect and annotate dialectal data [1, 24, 47, 48, 69, 76, 89, 164, 169, 186. The LDC was first to create a Levantine and an Egyptian Arabic Treebanks [129.

In the Arab world, the efforts are relatively limited in terms of creating annotated corpora. Examples include BZU's Curras, the Palestinian Arabic annotated corpus [17], NYUAD's Gumar, the Emirati Arabic annotated corpus [120, and Al-Mus'haf Quranic Arabic corpus [209, which is based on the Quran and includes morpho-syntactic annotations such as stems, stem patterns, and roots. Another annotation effort with a focus on MSA spelling and grammar correction is the Qatar Arabic Language Bank (QALB), which was developed between Columbia and CMUQ 201]. Other specialized annotated corpora developed in the Arab world include NYUAD's parallel gender corpus with sentences in masculine and feminine for anti-gender bias research, the Arab-Acquis corpus pairing Arabic with all of Europe's languages for a portion of European parliamentary proceedings, and the MADAR corpus of parallel dialects created in collaboration with CMUQ.

In contrast to annotated corpora, there are many unannotated datasets. Most large data sets also started outside the Arab world, e.g., the Agence France Press document collection, which is heavily used for Arabic information retrieval evaluation, the LDC's Arabic Gigaword [155], Arabic Wikipedia, and the ArTenTen corpus [36]. Important collections in the Arab World include: the International Corpus of Arabic (Bibliotheca Alexandrina); Shamela, 
a large-scale corpus (1B words) covering the past 14 centuries of Arabic 1 ; the Tashkeela corpus containing 75M fully vocalized words (National Computer Science Engineering School in Algeria) [210]; NYUAD's Gumar Gulf Arabic corpus containing over 100M words of internet novels [119]; and Abu El-Khair corpus (Umm Al-Qura University, KSA) [78. The success of word embedding models [137, 157] trained on unannotated data and resulting in improved performance for NLP tasks with little or no feature engineering have led to many contributions in Arabic NLP [19, 88, 189. The more recent appearance of contextualized embeddings trained on unannotated data, such as BERT [67], is creating promising possibilities for improving many Arabic NLP tasks. At the time of writing this article, a handful of contextualized embedding models are known to support Arabic including Multilingual BERT [68, Arabic BERT [166, AraBERT (AUB) [35, GigaBert (Ohio State U.) [125], Marbert (U. of British Columbia) [8], and QARiB (QCRI).

Lexical Resources We can distinguish three types of lexical resources (aka, lexicons, dictionaries and databases): (a) morphological resources that encode all inflected forms of words, (b) lexical resources that are lemma based, such as machine readable monolingual and multilingual dictionaries, and (c) semantic resources that link lemmas to each other, such as wordnets and ontologies. These resources are useful for a variety of NLP tasks.

Some of the earliest publicly available Arabic lexical resources were created outside of the Arab world in the second wave mentioned earlier. The Buckwalter Arabic Morphological Analyzer (BAMA), with its extended version called Standard Arabic Morphological Analyzer (SAMA), both available from the LDC, provided one of the first stem databases with tags and morphological solutions, and are used in a number of tools. Elixir-FM is a functional morphology analyzer developed in Charles University in Czech Republic 187. The DIINAR 71 Arabic morphological database is a full form resource developed in France. The Tharwa lemma-based lexicon was developed at Columbia University and included 70k entries in Egyptian Arabic, MSA and English; and later extended with Levantine Arabic [70. Arabic WordNet is a semantic lexicon consisting of about 11k synsets with subset and superset relationships between concepts and linked to a number of other languages through the Global Wordnet effort. This effort was done by a number of American and European universities [82. And the Arabic VerbNet 140] classifies verbs that have the same syntactic descriptions and argument structure (U. of Konstanz, Germany).

Some of the efforts in the Arab world led to multiple notable resources. Al-Khalil morphological analyzer is a large morphological database for Arabic developed by researchers in Morocco and Qatar [50] Calima Star is an extension of the BAMA/SAMA family done at NYUAD and is part of the CAMeL Tools toolkit [151, 195. BZU developed a large Arabic lexicographic database constructed from 150 lexicons that are diacritized and standardized [115. The MADAR project (NYUAD and CMUQ) includes a lexicon with 47k lemma

${ }^{1}$ https://shamela.ws/ 
entries covering 25 city dialects in Parallel [48. Other lexicons have been developed for Algerian [9, Tunisian [176], and Morrocan [191. Finally, in terms of semantic lexical resources, the BZU Arabic Ontology [114] is a formal Arabic wordnet with more than $20 \mathrm{k}$ concepts that was built with ontological analysis in mind and is linked to the Arabic Lexicographic Database, Wikidata, and other resources [116].

More Arabic resources can be found in known international repositories (namely ELRA/ELDA, LDC, and CLARIN) or directly from their authors' websites [87, 102, 198. Unfortunately, many are not interoperable, have been built using different tools and assumptions, released under propriety licenses, and a few are comprehensive. Serious, well-planned, and well-coordinated investment in resources will be instrumental for the future of Arabic NLP.

\subsubsection{Morphological Processing}

Given the challenges of Arabic morphological richness and ambiguity, morphological processing has received a lot of attention. The task of morphological analysis refers to the generation of all possible readings of a particular undiacritized word out of context. Morphological disambiguation is about identifying the correct in-context reading. This broad definition allows us to think of wordlevel tasks such as Part-of-Speech (POS) tagging, stemming, diacritization and tokenization as sub-types of morphological disambiguation that focus on specific aspects of ambiguity.

Most work on Arabic morphological analysis and disambiguation is on MSA; however there is a growing number of efforts on dialectal Arabic [64, 123, 156, 174, 204. There are a number of commonly used morphological analyzers for Standard and dialectal Arabic (Egyptian and Gulf), e.g., BAMA, SAMA, ElixirFM, Al-Khalil, Calima Egyptian, and CAlima Star [50, 51, 91, 96, 121, 187. Some of the morphological disambiguation systems disambiguate the analyses that are produced by a morphological analyzer using PATB as a training corpus, e.g., MADAMIRA (initially developed at Columbia U.), and other variants of it from NYUAD [122, 151, 156, 205. Farasa (from QCRI) uses independent models for tokenization [1] and POS tagging [63].

\subsubsection{Syntactic Processing}

Syntactic parsing is the process of generating a parse tree representation for a sentence that indicates the relationship among its words. For example, a

syntactic parse of the sentence قرَأ الطَالبُ المتًابَ الحبديَ'] read the-student the-book the-new; the student read the new book' would indicate that the adjective the-new modifies the noun the-book, which itself is the direct object of the verb read.

There are many syntactic representations. Most commonly used in Arabic are the PATB constituency representation [128, the CATiB dependency representation [99], and the Universal Dependency (UD) representation [194]. All of these were developed outside of the Arab world. The UD representation is an 
international effort, where NYUAD is the representative of the Arab world on Arabic.

The most popular syntactic parsers for Arabic are: Stanford, Farasa (QCRI), and CamelParser (NYUAD). Stanford is a statistical parser from the Stanford Natural Language Processing Group that can parse English, German, Arabic and Chinese. For Arabic, it uses a probabilistic context free grammar that was developed based on PATB [92. Updated versions of the parser use a shift-reduce algorithm [212] and neural transition-based dependency parsing [54]. Farasa is an Arabic NLP toolkit that provides syntactic constituency and dependency parsing 211. CamelParser is a dependency parser trained on CATiB treebank using MaltParser [148, a language-independent and data-driven dependency parser 183. A discussion and survey of some of the Arabic parsing work is presented in [102].

\subsubsection{Named Entity Recognition}

Named Entity recognition (NER) is the task of identifying one or more consecutive words in text that refer to objects that exist in the real-world (named entities), such as organizations, persons, locations, brands, products, foods, etc. NER is essential for extracting structured data from an unstructured text, relationship extraction, ontology population, classification, machine translation, question answering, and other applications. Among the challenges facing Arabic NER compared to English NER is the lack of letter casing which strongly helps English NER and the high degree of ambiguity, including especially confusable proper names and adjectives, e.g. كر Kariym can be the name 'Kareem' or the adjective 'generous'.

Arabic NER approaches include the use of hand-crafted heuristics, machine learning, and hybrids of both with heavy reliance on gazetteers [44, 55, 177]. Recent approaches exploited cross-lingual links between Arabic and English knowledge bases to expand Arabic gazetteers and to carry over capitalization from English to Arabic [55. Much of the earlier work on Arabic NER focused on formal text, typically written in MSA. However, applying models trained on MSA text to social media (mostly dialectal) text has led to unsatisfactory results 59. Recent contextualized embeddings and other deep learning approaches such as sequence to sequence models and convolutional neural networks have led to improved results for Arabic NER [28, 118, 126, 151]. It is expected that the use of contextualized embeddings trained on larger corpora of varying Arabic dialects, coupled with the use of deep learning models is likely to contribute positively to Arabic NER. As with other utilities, early research was done outside of the Arab world, but more work is now happening in the Arab world. A extensive list of Arabic NER challenges and solutions can be found in 177.

\subsubsection{Dialect Identification}

Dialect identification (DID) is the task of automatically identifying the dialect of a particular segment of speech or text of any size (i.e., word, sentence, or 
document). This task has been attracting increasing attention in NLP for a number of language varieties [207. DID has been shown to be important for several NLP tasks where prior knowledge about the dialect of an input text can be helpful, such as machine translation [172, sentiment analysis [23, and author profiling [164.

Early Arabic multi-dialectal data sets and models focused on the regional level [45, 47, 66, 81, 93, 136, 203. The Multi Arabic Dialects Application and Resources (MADAR) project aimed to create a finer grained dialectal corpus and lexicon [48. The data was used for dialectal identification at the city level 150, 170 of 25 Arab cities, and was used in a shared task for DID [49]. The main issue with that data is that it was commissioned and not naturally occurring. Concurrently, larger Twitter-based datasets covering 10-21 countries were also introduced [3, 4, 144, 199. The Nuanced Arabic Dialect Identification (NADI) Shared Task [7 followed earlier pioneering works by providing country-level dialect data for 21 Arab countries, and introduced a province-level identification task aiming at exploring a total of 100 provinces across these countries. Also here, earlier efforts started in the west, most notably work in Johns Hopkins University [202, but more work is happening now in the Arab world (NYUAD and QCRI) [3, 49].

\subsubsection{Infrastructure}

To aid the development of NLP systems, a number of multi-lingual infrastructure toolkits have been developed, e.g., GATE2 2 , Stanford CoreNLP 3 and UIMA 4 . Their philosophy is to gather and develop several NLP tools within a single and homogeneous structure that is flexible, extensible and modular. They offer researchers easy access to several tools through command-line interfaces (CLIs) and application programming interfaces (APIs), thus eliminating the need to develop them from scratch every time. While Arabic NLP has made significant progress with the development of several enabling tools, such as POS taggers, morphological analyzers, text classifiers, and syntactic parsers, there is a limited number of homogeneous and flexible Arabic infrastructure toolkits that gather these components. MADAMIRA [156] is a Java-based system providing solutions to fundamental NLP tasks for Standard and Egyptian Arabic. These tasks include diacritization, lemmatization, morphological analysis and disambiguation, POS tagging, stemming, glossing, (configurable) tokenization, basephrase chunking and NER 5 MADAMIRA's signature approach is to address multiple morphology related tasks together in one fell swoop producing deep linguistic representations. Farasa 6 is a collection of Java libraries and CLIs for MSA. These include separate tools for diacritization [58, 143, segmentation 1], lemmatization [141], POS tagging [63], parsing [211, and NER [56]. SAFAR] is

\footnotetext{
${ }^{2}$ https://gate.ac.uk

3 https://stanfordnlp.github.io/CoreNLP/

4 https://uima.apache.org/d/uimaj-current/

${ }^{5}$ https://camel.abudhabi.nyu.edu/madamira/

6 http://qatsdemo.cloudapp.net/farasa/

7 http://arabic.emi.ac.ma/safar/
} 
a Java-based framework bringing together all layers of Arabic NLP: resources, pre-processing, morphology, syntax, and semantics. CAMeL Tools 151 is a recently developed collection of open-source tools, developed in Python, that supports both MSA and Arabic dialects 8 It currently provides APIs and CLIs for pre-processing, morphological modeling, dialect identification, NER, and sentiment analysis. Other notable efforts include AraNLP [32, ArabiTools 9 and Adawat 10 A feature comparison of some Arabic infrastructures can be found in 151] while a detailed survey and a software engineering comparative study can be found in [113. Whatever the specific choice a researcher might make, the trend to use infrastructures more extensively will significantly save time and change the way Arabic applications are designed and developed.

\subsection{Arabic NLP Applications}

\subsubsection{Machine Translation}

Machine Translation (MT) is one of the earliest and most worked on areas in NLP. The task is to map input text in a source language such as English to an output text in a target language such as Arabic. Early MT research was heavily rule-based 194, 109, 111, 159, 179; ; however now it is almost completely corpus-based using a range of statistical and deep learning models, depending on resource availability.

For MSA, parallel data in the news domain is plentiful 11 There are other large Arabic parallel collections under the OPUS project [196] and as part of the UN corpus [158. Other specialized corpora include the Arab-Acquis corpus pairing with European languages (NYUAD) 101] and the AMARA educational domain parallel corpus (QCRI) 2. Dialectal parallel data are harder to come by and most are commissioned translations [47, 48, 85, 136, 208.

There are many other efforts in Statistical MT (SMT) from and to Arabic [52, 79, 83, 100, 104, 108, 147, 192. Recently, Deep Neural Networks have been adopted for Arabic Machine Translation as in [15, 29, 33, 90, 154. While most researched MT systems for Arabic target English, there have been efforts on MT for Arabic and other languages, e.g., Chinese [97, Russian [206], Japanese 112, and all of the European Union languages [101].

MT for Arabic dialects is more difficult due to limited resources, but there are noteworthy efforts exploiting similarities between MSA and dialects in universities and research group around the world [167, 168, 173, 175, 178. Finally, there is a notable effort on Arabic sign-language translation at King Fahd University of Petroleum and Minerals [127. For recent surveys of Arabic MT, see 34. Despite all these contributions, much research work is still needed to improve the performance of Machine translation for Arabic.

\footnotetext{
${ }^{8}$ https://github.com/CAMeL-Lab

https://www.arabitools.com/

10 http://adawat.sourceforge.net/

${ }^{11}$ Linguistic Data Consortium (LDC) resources: LDC2004T18, LDC2004T14, and LDC2007T08.
} 


\subsubsection{Pedagogical Applications}

Pedagogical applications (PA) focus on building tools to develop or model four major skills: reading, writing, listening, and speaking. Arabic PA research has solely focused on MSA. PA systems can be distinguished in terms of their target learners as first language (L1) or second (foreign) language (L2) systems. This distinction can be problematic since, for Arabs, learning to read MSA is somewhat akin to reading a foreign tongue due to its lexical and syntactic divergence from native dialects. We focus our Arabic PA discussion on (a) computerassisted language learning (CALL) systems, (b) readability assessment, and (c) resource-building efforts.

CALL systems utilize NLP enabling technologies to assist language learners. There has been a number of efforts in Arabic CALL exploring a range of resources and techniques. Examples include the use of Arabic grammar and linguistic analysis rules to help learners identify and correct a variety of errors 180, 181; and multi-agent tutoring systems that simulate the instructor, the student, the learning strategy, and include a log book to monitor progress, and a learning interface [133, 134. Another approach focuses on enriching the reading experience with concordances, text-to-speech (TTS), morpho-syntactic analysis, and auto-generated quiz questions [130.

Readability Assessment is the task of automatic identification of a text's readability, i.e., its ability to be read and understood by its reader employing an acceptable amount of time and effort. There has been a range of approaches for Arabic L1 and L2 readability. On one end, we find formulas using languageindependent variables such as text length, average word length, and average sentence length, number of syllables in words, the relative rarity or absence of dialectal alternatives, and the presence of less common letters [53, 77]. Others integrate Arabic morphological, lexical and syntactic features with supervised machine learning approaches [16, 22, 165].

Although some progress has been made for both L1 and L2 PA, the dearth of resources compared with English remains the bottleneck for future progress. Resource-building efforts have focused on L1 readers with particular emphasis on grade school curricula [12, 16, 22. There is a push to inform the enhancement of curricula using pedagogical tools and to compare curricula across Arab countries [21, 103]. The L2 PAs are even more limited with limited corpora 130. with disproportionate focus on beginners 12 There is a definite need for augmenting these corpora in a reasoned way, taking into consideration different text features and learners, both young and old, beefing up the sparsely populated levels with authentic material, and exploiting technologies such as text simplification and text error analysis and correction. Learner corpora, which as the name suggests are produced by learners of Arabic can inform the creation of tools and corpora [26, 163, 200]. A recent effort developed a large-scale Arabic readability lexicon compatible with an existing morphological analysis system 17.

\footnotetext{
${ }^{12}$ https://learning.aljazeera.net/en
} 


\subsubsection{Information Retrieval and Question Answering}

With the increasing volume of Arabic content, information retrieval (aka search) has become a necessity for many domains such as medical records, digital libraries, web content, and news. The main research interests have focused on retrieval of formal language, mostly in the news domain, with ad hoc retrieval [149, OCR document retrieval [60, 65], and cross-language retrieval [80. The literature on other aspects of retrieval continues to be sparse or non-existent, though some of these aspects have been investigated by industry. Others aspects of Arabic retrieval that have received some attention include document image retrieval [131], speech search [153, social media 62, 107] and web search 105, 190, and filtering [61, 132. However, efforts on different aspects of Arabic retrieval continue to be deficient and severely lacking behind efforts in other languages. Examples of unexplored problems include searching Wikipedia, which contains semi-structured content, religious text [135, which often contain semistructured data such chains of narrations, rulings, and commentaries, Arabic forums, which are very popular in the Arab world and constitute a significant portion of the Arabic web, and poetry. To properly develop algorithms and methods to retrieve such content, standard test sets and clear usage scenarios are required. We expect that recent improvements in contextual embeddings can positively impact the effectiveness of many retrieval tasks.

Another IR related problem is question answering, which comes in many flavors, the most common of which is attempting to identify a passage or a sentence that answers a question 106. Performing such a task may employ a large set of NLP tools such as parsing, NER, co-reference resolution, and text semantic representation. There has been limited research on this problem [13, 161] and existing commercial solutions such as Ujeeb.com are rudimentary.

\subsubsection{Dialogue Systems}

Automated dialog systems capable of sustaining a smooth and natural conversation with users have attracted considerable interest from both research and industry in the past few years. This technology is changing how companies engage with their customers among many other applications. While commercial dialog systems by big multinational companies such as Amazon Alexa, Google Home, and Apple Siri support many languages, only Apple Siri supports Arabic with limited performance. There are some strong recent competitors in the Arab world, particularly Arabot13 and Mawdoo3's Salma 14

While there is an important growing body of research on English language dialog systems, current NLP methods for Arabic language dialogue are mostly based on handcrafted rule-based systems and methods that use feature engineering 27. Among the earliest research efforts on Arabic dialog applications is the Quran chatbot 184, where the conversation length is short since the system answers a user input with a single response. It uses a retrieval-based

\footnotetext{
13 https://arabot.io/

${ }^{14}$ http://salma.ai/
} 
model as the dataset is limited by the content of the Quran. A recent approach used deep learning techniques for text classification and NER to build a natural language understanding module - the core component of any dialogue system for the domain of home automation in Arabic [43. A unique dialogue system from NYUAD explored bilingual interfaces where Arabic speech can be used as input to an English bot that displays Arabic subtitles [10]. Other works have focused on developing dialog systems for the case of Arabic dialects, e.g. the publicly available NYUAD Egyptian dialect chatbot Botta, and KSU's Saudi dialect Information Technology focused chatbot Nabiha [14].

\subsubsection{Sentiment and Emotion Analysis}

Sentiment analysis (SA), aka opinion mining, is the task of identifying the affective states and subjective information in a text. For example, an Egyptian Arabic movie review such as احسن فيلم السنة دي! 'the best movie this year!' is said to indicate a positive sentiment. SA is a very powerful tool for tracking customer satisfaction, carrying out competition analysis, and generally gauging public opinion towards a specific issue, topic, or product. SA has attracted a lot of attention in the Arabic research community during the last decade, connected with the availability of large volumes of opinionated and sentiment reflecting data from Arabic social media. Early Arabic SA efforts focused on the creation of needed resources such as sentiment lexicons, training datasets, and sentiment treebanks [5, 38, 41, 74, 84, 86, 124, 139, 160, 171, 185, as well as shared task benchmarks [138, 162. Arabic SA solutions span a range of methods from the now conventional use of rules and lexicons [6, 38, 75] to machine learning based methods [11, 39, 41, as well as hybrid approaches employing morphological and syntactic features 20]. Recently, fine-tuning large pre-trained language models has achieved improved Arabic SA results [8, 35]. Arabic emotion recognition is a closely related topic that has attracted some attention recently. It aims to identify a variety of emotions in text such as anger, disgust, surprise, and joy 31, 40, 42, 110, 182. Similar to how SA resources and models started maturing, a lot of work still needs to be done in emotion recognition. Another related problem is stance detection, which attempts to identify positions expressed on a topic or towards an entity. Stances are often expressed using non-sentiment words [46]. For a recent comprehensive survey on the status of Arabic SA and the future directions, see [37].

\subsubsection{Content Moderation on Social Media}

The task of content moderation is about the enforcement of online outlets' policies against posting user comments that contain offensive language, hate speech, cyber-bullying, and spam among other types of inappropriate or dangerous content 15 Such content cannot be easily detected given the huge volume of posts, dialectal variations, creative spelling on social media, and the scarcity of available data and detection tools. This area is relatively new for Arabic. One of

\footnotetext{
${ }^{15}$ https://www.bbc.co.uk/usingthebbc/terms/what-are-the-rules-for-commenting/
} 
the more active areas has to do with the detection of offensive language, which covers targeted attacks, vulgar and pornographic language, and hate speech. Initial work was performed on comments from a news site and limited numbers of tweets [145] and YouTube comments [25. Some works focused on adult content [30] and others on hate speech. Recent benchmarking shared tasks included the automatic detection of such language in Twitter domain [146. Work on spam detection on Twitter is nascent and much work is required [142].

\section{Future Outlook}

Arabic NLP has many challenges, but it has also seen many successes and developments over the last 40 years. We are optimistic by its continuously positive albeit (sometimes) slow development trajectory. For the next decade or two, we expect a large growth in the Arabic NLP market. This is consistent with the global rising demands and expectations for language technologies and the increase in NLP research and development in the Arab world. The growing number of researchers and developers working on NLP in the Arab world makes it a very fertile ground ready for major breakthroughs. To support this vision, we believe it is time to have an association for Arabic language technologists that brings together talent and resources, and sets standards for the Arabic NLP community. Such an organization can support NLP education in the Arab world, serve as a hub for resources, and advocate for educators and researchers in changing old-fashioned university policies regarding journal-focused evaluation, and encouraging collaborations within the Arab world by connecting academic, industry, and governmental stakeholders. We also recommend more open source tools and public data are made available to create a basic development framework that lowers the threshold for joining the community, thus attracting more talent that will form the base of the next generation of Arabic NLP researchers, developers, and entrepreneurs.

\section{References}

[1] Abdelali, A., Darwish, K., Durrani, N., and Mubarak, H. Farasa: A Fast and Furious Segmenter for Arabic. In Proceedings of the Conference of the North American Chapter of the Association for Computational Linguistics (NAACL) (San Diego, California, 2016), pp. 11-16.

[2] Abdelali, A., Guzman, F., Sajuad, H., and Vogel, S. The Amara Corpus: Building parallel language resources for the educational domain. In Proceedings of the Language Resources and Evaluation Conference (LREC) (Reykjavik, Iceland, 2014).

[3] Abdelali, A., Mubarak, H., Samin, Y., Hassan, S., and DarWISH, K. Arabic dialect identification in the wild. arXiv preprint arXiv:2005.06557 (2020). 
[4] Abdul-Mageed, M., Alhuzali, H., and Elaraby, M. You tweet what you speak: A city-level dataset of Arabic dialects. In Proceedings of the Language Resources and Evaluation Conference (LREC) (Miyazaki, Japan, 2018).

[5] Abdul-Mageed, M., And Diab, M. Toward Building a Large-Scale Arabic Sentiment Lexicon. In Proceedings of the 6th International Global WordNet Conference (Matuse, Japan, 2012), pp. 18-22.

[6] Abdul-Mageed, M., And Diab, M. SANA: A large scale multigenre, multi-dialect lexicon for Arabic subjectivity and sentiment analysis. In Proceedings of the Language Resources and Evaluation Conference (LREC) (Reykjavik, Iceland, 2014), pp. 1162-1169.

[7] Abdul-Mageed, M., Zhang, C., Bouamor, H., and Habash, N. NADI 2020: The First Nuanced Arabic Dialect Identification Shared Task. In Proceedings of the Fifth Arabic Natural Language Processing Workshop (WANLP 2020) (Barcelona, Spain, 2020).

[8] Abdul-Mageed, M., Zhang, C., Elmadany, A., and Ungar, L. Toward micro-dialect identification in diaglossic and code-switched environments, 2020.

[9] Abidi, K., And SmaïLI, K. An automatic learning of an algerian dialect lexicon by using multilingual word embeddings. In 11th edition of the Language Resources and Evaluation Conference, LREC 2018 (2018).

[10] Abu Ali, D., Ahmad, M., Al Hassan, H., Dozsa, P., Hu, M., VARIAS, J., AND HABASH, N. A bilingual interactive human avatar dialogue system. In Proceedings of the 19th Annual SIGdial Meeting on Discourse and Dialogue (Melbourne, Australia, July 2018), Association for Computational Linguistics, pp. 241-244.

[11] Abu Farha, I., And Magdy, W. Mazajak: An online Arabic sentiment analyser. In Proceedings of the Fourth Arabic Natural Language Processing Workshop (Florence, Italy, Aug. 2019), Association for Computational Linguistics, pp. 192-198.

[12] Al-Ajlan, A. A., Al-Khalifa, H. S., and Al-Salman, A. S. Towards the development of an automatic readability measurements for Arabic language. In 2008 Third International Conference on Digital Information Management (2008), pp. 506-511.

[13] Al-Bataineh, H., Farhan, W., Mustafa, A., Seelawi, H., and T. Al-Natshen, H. Deep contextualized pairwise semantic similarity for Arabic language questions. In 2019 IEEE 31st International Conference on Tools with Artificial Intelligence (ICTAI) (2019), pp. 1586-1591. 
[14] Al-Ghadhban, D., And Al-Twairesh, N. Nabiha: An Arabic dialect chatbot. International Journal of Advanced Computer Science and Applications 11, 3 (2020).

[15] Al-Ibrahim, R., and Duwairi, R. M. Neural machine translation from jordanian dialect to modern standard Arabic. In 2020 11th International Conference on Information and Communication Systems (ICICS) (2020), IEEE, pp. 173-178.

[16] Al-Khalifa, H., And Al-Ajlan, A. A. Automatic readability measurements of the Arabic text: An exploratory study. ARABIAN JOURNAL FOR SCIENCE AND ENGINEERING 35, 2 (2010), 103-124.

[17] Al Khalil, M., Habash, N., And Jiang, Z. A large-scale leveled readability lexicon for Standard Arabic. In Proceedings of the 12th Language Resources and Evaluation Conference (Marseille, France, May 2020), European Language Resources Association, pp. 3053-3062.

[18] Al-Sabbagh, R., And Girju, R. YADAC: Yet another Dialectal Arabic Corpus. In Proceedings of the Eighth International Conference on Language Resources and Evaluation (LREC-2012) (2012), pp. 2882-2889.

[19] Al Sallab, A., Haju, H., Badaro, G., Baly, R., El-Haju, W., and ShabAN, K. Deep learning models for sentiment analysis in Arabic. In Proceedings of the second workshop on Arabic natural language processing (2015), pp. 9-17.

[20] Al-Smadi, M., Al-Ayyoub, M., Jararweh, Y., And Qawasmeh, O. Enhancing aspect-based sentiment analysis of Arabic hotels' reviews using morphological, syntactic and semantic features. Information Processing \& Management 56, 2 (2019), 308-319.

[21] Al-Sulaiti, L., Abbas, N., Brierley, C., Atwell, E., And Alghamdi, A. Compilation of an Arabic children's corpus. In Proceedings of the Tenth International Conference on Language Resources and Evaluation (LREC'16) (Portorož, Slovenia, May 2016), European Language Resources Association (ELRA), pp. 1808-1812.

[22] Al Tamimi, A.-K., Jaradat, M., AlJarrah, N., and Ghanem, S. Aari: automatic Arabic readability index. International Arab Journal of Information Technology (IAJIT) 11, 4 (2014), 370-378.

[23] Al-Twairesh, N., Al-Khalifa, H., and AlSalman, A. AraSenTi: Large-Scale Twitter-Specific Arabic Sentiment Lexicons. In Proceedings of the 54th Annual Meeting of the Association for Computational Linguistics (Berlin, Germany, 2016).

[24] Al-Twairesh, N., Al-Matham, R., Madi, N., Almugren, N., AlAljmi, A.-H., Alshalan, S., Alshalan, R., Alrumayyan, N., 
Al-Manea, S., Bawazeer, S., Al-Mutlaq, N., Almanea, N., Huwaymil, W. B., Alqusair, D., Alotaibi, R., Al-Senaydi, S., and Alfutamani, A. SUAR: Towards building a corpus for the Saudi dialect. In Proceedings of the International Conference on Arabic Computational Linguistics (ACLing) (2018).

[25] Alakrot, A., Murray, L., And Nikolov, N. S. Towards accurate detection of offensive language in online communication in Arabic. Procedia computer science 142 (2018), 315-320.

[26] Alfaifi, A., Atwell, E., And Ibraheem, H. Arabic learner corpus (alc) v2 : A new written and spoken corpus of Arabic learners, 2014.

[27] AlHumoud, S., Al Wazrah, A., and Aldamegh, W. Arabic chatbots: A survey. INTERNATIONAL JOURNAL OF ADVANCED COMPUTER SCIENCE AND APPLICATIONS 9, 8 (2018), 535-541.

[28] Ali, M., Tan, G., and Hussain, A. Boosting Arabic named-entity recognition with multi-attention layer. IEEE Access 7 (2019), 4657546582 .

[29] Almahairi, A., Cho, K., Habash, N., And Courville, A. First Result on Arabic Neural Machine Translation. arXiv e-prints (June 2016), arXiv:1606.02680.

[30] Alshehri, A., Nagoudi, E. M. B., Alhuzali, H., And AbdulMAgeed, M. Think before your click: Data and models for adult content in Arabic twitter. In The 2nd Text Analytics for Cybersecurity and Online Safety Workshop (TA-COS-2018) (2018).

[31] Alswaidan, N., And Menai, M. E. B. Hybrid feature model for emotion recognition in Arabic text. IEEE Access 8 (2020), 37843-37854.

[32] Althobaiti, M., Kruschwitz, U., And Poesio, M. Aranlp: A javabased library for the processing of Arabic text. In Lrec (2014).

[33] Ameur, M. S. H., Guessoum, A., And Meziane, F. Improving Arabic neural machine translation via n-best list re-ranking. Mach. Transl. 33, 4 (2019), 279-314.

[34] Ameur, M. S. H., Meziane, F., and Guessoum, A. Arabic machine translation: A survey of the latest trends and challenges. Computer Science Review 38 (2020), 100305.

[35] Antoun, W., Baly, F., And Haju, H. Arabert: Transformerbased model for Arabic language understanding. arXiv preprint arXiv:2003.00104 (2020). 
[36] Arts, T., Belinkov, Y., Habash, N., Kilgarriff, A., and SuCHOMEL, V. artenten: Arabic corpus and word sketches. Journal of King Saud University-Computer and Information Sciences 26, 4 (2014), $357-371$.

[37] Badaro, G., Baly, R., Haju, H., El-Haju, W., Shaban, K. B., Habash, N., Al-Sallab, A., ANd Hamdi, A. A survey of opinion mining in Arabic: a comprehensive system perspective covering challenges and advances in tools, resources, models, applications, and visualizations. ACM Transactions on Asian and Low-Resource Language Information Processing (TALLIP) 18, 3 (2019), 1-52.

[38] Badaro, G., Baly, R., Haju, H., Habash, N., and El-Haju, W. A large scale Arabic sentiment lexicon for Arabic opinion mining. In Proceedings of the Workshop for Arabic Natural Language Processing (WANLP) (Doha, Qatar, 2014), pp. 165-173.

[39] Badaro, G., El Jundi, O., Khaddaj, A., Maarouf, A., Kain, R., HajJ, H., And El-HaJj, W. EMA at SemEval-2018 task 1: Emotion mining for Arabic. In Proceedings of The 12th International Workshop on Semantic Evaluation (New Orleans, Louisiana, June 2018), Association for Computational Linguistics, pp. 236-244.

[40] Badaro, G., Jundi, H., Haju, H., El-Haju, W., and Habash, N. Arsel: A large scale Arabic sentiment and emotion lexicon. In OSACT 3 : The 3rd Workshop on Open-Source Arabic Corpora and Processing Tools (2018), p. 26.

[41] Baly, R., Haju, H., Habash, N., Shaban, K. B., and El-Haju, W. A sentiment treebank and morphologically enriched recursive deep models for effective sentiment analysis in Arabic. ACM Transactions on Asian and Low-Resource Language Information Processing (TALLIP) 16, 4 (2017), 1-21.

[42] Baly, R., Khaddaj, A., Haju, H., El-Haju, W., and Shaban, K. B. Arsentd-lev: A multi-topic corpus for target-based sentiment analysis in Arabic levantine tweets. arXiv preprint arXiv:1906.01830 (2019).

[43] Bashir, A. M., Hassan, A., Rosman, B., Duma, D., and Ahmed, M. Implementation of a neural natural language understanding component for Arabic dialogue systems. Procedia computer science 142 (2018), 222-229.

[44] Benajiba, Y., Diab, M., And Rosso, P. Arabic Named Entity Recognition using Optimized Feature Sets. In Proceedings of the Conference on Empirical Methods in Natural Language Processing (EMNLP) (Honolulu, Hawaii, 2008), pp. 284-293.

[45] Biadsy, F., And Hirschberg, J. Using Prosody and Phonotactics in Arabic Dialect Identification. In Proceedings of the Conference of the 
International Speech Communication Association (Interspeech) (Brighton, UK, 2009).

[46] Borge-Holthoefer, J., Magdy, W., Darwish, K., And Weber, I. Content and network dynamics behind egyptian political polarization on twitter. In Proceedings of the 18th ACM Conference on Computer Supported Cooperative Work $E$ Social Computing (2015), pp. 700-711.

[47] Bouamor, H., Habash, N., And Oflazer, K. A multidialectal parallel corpus of Arabic. In Proceedings of the Language Resources and Evaluation Conference (LREC) (Reykjavik, Iceland, 2014).

[48] Bouamor, H., Habash, N., Salameh, M., Zaghouani, W., RamBow, O., Abdulrahim, D., Obeid, O., Khalifa, S., Eryani, F., Erdmann, A., And Oflazer, K. The MADAR Arabic Dialect Corpus and Lexicon. In Proceedings of the Language Resources and Evaluation Conference (LREC) (Miyazaki, Japan, 2018).

[49] Bouamor, H., Hassan, S., and Habash, N. The MADAR shared task on Arabic fine-grained dialect identification. In Proceedings of the Fourth Arabic Natural Language Processing Workshop (2019), pp. 199-207.

[50] Boudchiche, M., Mazroui, A., Bebah, M. O. A. O., Lakhouaja, A., AND Boudlal, A. AlKhalil Morpho Sys 2: A robust Arabic morphosyntactic analyzer. Journal of King Saud University - Computer and Information Sciences 29, 2 (2017), 141-146.

[51] Buckwalter, T. Buckwalter Arabic morphological analyzer version 1.0. Linguistic Data Consortium (LDC) catalog number LDC2002L49, ISBN 1-58563-257-0, 2002.

[52] Carpuat, M., Marton, Y., and Habash, N. Improving Arabic-toenglish statistical machine translation by reordering post-verbal subjects for alignment. In Proceedings of the ACL 2010 Conference Short Papers (2010), pp. 178-183.

[53] Cavalli-Sforza, V., Saddiki, H., and Nassiri, N. Arabic readability research: Current state and future directions. Arabic Computational Linguistics (WASJ) 142 (2018), 38-49.

[54] Chen, D., And Manning, C. D. A fast and accurate dependency parser using neural networks. In Proceedings of the 2014 conference on empirical methods in natural language processing (EMNLP) (2014), pp. 740-750.

[55] Darwish, K. Named entity recognition using cross-lingual resources: Arabic as an example. In Proceedings of the 51st Annual Meeting of the Association for Computational Linguistics (Volume 1: Long Papers) (2013), pp. 1558-1567. 
[56] Darwish, K. Named entity recognition using cross-lingual resources: Arabic as an example. In Proceedings of the 51st Annual Meeting of the Association for Computational Linguistics (2013), pp. 1558-1567.

[57] Darwish, K. Arabizi Detection and Conversion to Arabic. In Proceedings of the Workshop for Arabic Natural Language Processing (WANLP) (Doha, Qatar, 2014), pp. 217-224.

[58] Darwish, K., Abdelali, A., Mubarak, H., and Eldesouki, M. Arabic diacritic recovery using a feature-rich bilstm model. arXiv preprint arXiv:2002.01207 (2020).

[59] Darwish, K., and Gao, W. Simple effective microblog named entity recognition: Arabic as an example. In LREC (2014), pp. 2513-2517.

[60] Darwish, K., AND Magdy, W. Error correction vs. query garbling for arabic ocr document retrieval. ACM Transactions on Information Systems (TOIS) 26, 1 (2007), 5-es.

[61] Darwish, K., And Magdy, W. Arabic information retrieval. Foundations and Trends in Information Retrieval 7, 4 (2014), 239-342.

[62] Darwish, K., Magdy, W., And Mourad, A. Language processing for Arabic microblog retrieval. In Proceedings of the 21st ACM international conference on Information and knowledge management (2012), pp. 24272430 .

[63] Darwish, K., Mubarak, H., Abdelali, A., and Eldesouki, M. Arabic pos tagging: Don't abandon feature engineering just yet. In Proceedings of the Third Arabic Natural Language Processing Workshop (2017), pp. 130-137.

[64] Darwish, K., Mubarak, H., Abdelali, A., Eldesouki, M., Samih, Y., Alharbi, R., Attia, M., Magdy, W., and Kallmeyer, L. Multidialect Arabic pos tagging: A CRF approach. In Proceedings of the Language Resources and Evaluation Conference (LREC) (Miyazaki, Japan, 2018).

[65] Darwish, K., And OARD, D. W. Term selection for searching printed arabic. In Proceedings of the 25th annual international ACM SIGIR conference on Research and development in information retrieval (2002), pp. 261-268.

[66] Darwish, K., Sajuad, H., And Mubarak, H. Verifiably Effective Arabic Dialect Identification. In Proceedings of the 2014 Conference on Empirical Methods in Natural Language Processing (EMNLP) (Doha, Qatar, 2014). 
[67] Devlin, J., Chang, M.-W., Lee, K., and Toutanova, K. Bert: Pretraining of deep bidirectional transformers for language understanding, 2018.

[68] Devlin, J., Chang, M.-W., Lee, K., and Toutanova, K. Bert: Pre-training of deep bidirectional transformers for language understanding. In Proceedings of the 2019 Conference of the North American Chapter of the Association for Computational Linguistics: Human Language Technologies, Volume 1 (Long and Short Papers) (Minneapolis, Minnesota, June 2019), Association for Computational Linguistics, pp. 4171-4186.

[69] Diab, M., Habash, N., Rambow, O., Altantawy, M., and BenaJIBA, Y. COLABA: Arabic dialect annotation and processing. In LREC workshop on Semitic language processing (2010), pp. 66-74.

[70] Diab, M. T., Al-Badrashiny, M., Aminian, M., Attia, M., Elfardy, H., Habash, N., Hawwari, A., Salloum, W., Dasigi, P., And Eskander, R. Tharwa: A Large Scale Dialectal Arabic-Standard Arabic-English Lexicon. In Proceedings of the Language Resources and Evaluation Conference (LREC) (Reykjavik, Iceland, 2014), pp. 37823789 .

[71] Dichy, J., And Hassoun, M. The dinar. 1 (dictionnaire informatisé de l'arabe, version 1) arabic lexical resource, an outline of contents and methodology. The ELRA news letter 10, 2 (2005).

[72] Dukes, K., And Buckwalter, T. A Dependency Treebank of the Quran using Traditional Arabic Grammar. In Proceedings of the Conference on Informatics and Systems (INFOS) (Cairo, Egypt, 2010).

[73] Dukes, K., And Habash, N. Morphological Annotation of Quranic Arabic. In Proceedings of the Language Resources and Evaluation Conference (LREC) (Valetta, Malta, 2010).

[74] El-Beltagy, S. R. NileUlex: A Phrase and Word Level Sentiment Lexicon for Egyptian and Modern Standard Arabic. In Proceedings of LREC 2016 (Portorož, Slovenia, 2016).

[75] El-Beltagy, S. R., And Ali, A. Open Issues in the Sentiment Analysis of Arabic Social Media : A Case Study. In Proceedings of 9th the International Conference on Innovations and Information Technology (IIT2013) (Al Ain, UAE, 2013).

[76] El-HaJ, M. Habibi - a multi dialect multi national Arabic song lyrics corpus. In Proceedings of the 12th Language Resources and Evaluation Conference (Marseille, France, May 2020), European Language Resources Association, pp. 1318-1326. 
[77] El-HaJ, M., AND RAYson, P. OSMAN - a novel Arabic readability metric. In Proceedings of the Tenth International Conference on Language Resources and Evaluation (LREC'16) (Portorož, Slovenia, May 2016), European Language Resources Association (ELRA), pp. 250-255.

[78] El-Khair, I. A. 1.5 billion words Arabic corpus. arXiv preprint arXiv:1611.04033 (2016).

[79] El Kholy, A., and Habash, N. Orthographic and morphological processing for English-Arabic statistical machine translation. In Proceedings of Traitement Automatique des Langues Naturelles (TALN) (Montréal, Canada, 2010). Montréal, Canada.

[80] Elayeb, B., And Bounhas, I. Arabic cross-language information retrieval: a review. ACM Transactions on Asian and Low-Resource Language Information Processing (TALLIP) 15, 3 (2016), 1-44.

[81] Elfardy, H., Al-Badrashiny, M., and Diab, M. Aida: Identifying code switching in informal Arabic text. In Proceedings of the Conference on Empirical Methods in Natural Language Processing (EMNLP) (Doha, Qatar, 2014), pp. 94-101.

[82] Elkateb, S., Black, W., Vossen, P., Farwell, D., Rodríguez, H., Pease, A., And Alkhalifa, M. Arabic wordnet and the challenges of Arabic. In Proceedings of Arabic NLP/MT Conference, London, UK (2006), pp. 665-670.

[83] Elming, J., and Habash, N. Syntactic reordering for english-Arabic phrase-based machine translation. In Proceedings of the EACL 2009 Workshop on Computational Approaches to Semitic Languages (2009), pp. 69-77.

[84] ElSahar, H., and El-Beltagy, S. R. Building Large Arabic Multidomain Resources for Sentiment Analysis. In Proceedings of CICLing 2015, Volume 9042 of the series Lecture Notes in Computer Science (2015), Springer Verlag, pp. 23-34.

[85] Erdmann, A., Habash, N., Taji, D., and Bouamor, H. Low Resourced Machine Translation via Morpho-syntactic Modeling: The Case of Dialectal Arabic. In Proceedings of the Machine Translation Summit (MT Summit) (2017).

[86] Eskander, R., and Rambow, O. SLSA: A Sentiment Lexicon for Standard Arabic. In Proceedings of the 2015 Conference on Empirical Methods in Natural Language Processing (2015), pp. 2545-2550.

[87] Farghaly, A., And Shatlan, K. Arabic natural language processing: Challenges and solutions. ACM Transactions on Asian Language Information Processing (TALIP) 8, 4 (2009), 1-22. 
[88] Farha, I. A., And Magdy, W. Mazajak: An online Arabic sentiment analyser. In Proceedings of the Fourth Arabic Natural Language Processing Workshop (2019), pp. 192-198.

[89] Gadalla, H., Kilany, H., Arram, H., Yacoub, A., El-Habashi, A., Shalaby, A., Karins, K., Rowson, E., MacIntyre, R., KingsBury, P., Graff, D., And McLemore, C. CAllhome Egyptian Arabic transcripts LDC97T19. Web Download. Philadelphia: Linguistic Data Consortium, 1997.

[90] Gashaw, I., and Shashirekha, H. L. Amharic-Arabic neural machine translation, 2019.

[91] Graff, D., Maamouri, M., Bouziri, B., Krouna, S., Kulick, S., AND BuCKWALter, T. Standard Arabic Morphological Analyzer (SAMA) Version 3.1, 2009. Linguistic Data Consortium LDC2009E73.

[92] Green, S., And Manning, C. D. Better Arabic Parsing: Baselines, Evaluations, and Analysis. In Proceedings of the International Conference on Computational Linguistics (COLING) (Beijing, China, 2010), pp. 394402.

[93] Guellil, I., And Azounou, F. Arabic Dialect Identification with an Unsupervised Learning (Based on a Lexicon). Application Case: ALGERIAN Dialect. In 2016 IEEE International Conference on Computational Science and Engineering (CSE) and IEEE Intl Conference on Embedded and Ubiquitous Computing (EUC) and 15th Intl Symposium on Distributed Computing and Applications for Business Engineering (DCABES) (2016), pp. $724-731$.

[94] Guessoum, A., And Zantout, R. A methodology for a semi-automatic evaluation of the lexicons of machine translation systems. Machine translation 16, 2 (2001), 127-149.

[95] Habash, N., Eryani, F., Khalifa, S., Rambow, O., Abdulrahim, D., Erdmann, A., Faraj, R., Zaghouani, W., Bouamor, H., Zalmout, N., Hassan, S., shargi, F. A., Alkhereyf, S., AbdulkaReem, B., Eskander, R., Salameh, M., and Saddiki, H. Unified guidelines and resources for Arabic dialect orthography. In Proceedings of the Language Resources and Evaluation Conference (LREC) (Miyazaki, Japan, 2018).

[96] Habash, N., Eskander, R., And Hawwari, A. A Morphological Analyzer for Egyptian Arabic. In Proceedings of the Workshop of the Special Interest Group on Computational Morphology and Phonology (SIGMORPHON) (Montréal, Canada, 2012), pp. 1-9.

[97] Habash, N., and Hu, J. Improving Arabic-chinese statistical machine translation using english as pivot language. In Proceedings of the Fourth Workshop on Statistical Machine Translation (2009), pp. 173-181. 
[98] Habash, N., and Rambow, O. Arabic tokenization, part-of-speech tagging and morphological disambiguation in one fell swoop. In Proceedings of the Conference of the Association for Computational Linguistics (ACL) (Ann Arbor, Michigan, 2005), pp. 573-580.

[99] Habash, N., and Roth, R. CATiB: The Columbia Arabic Treebank. In Proceedings of the Joint Conference of the Association for Computational Linguistics and the International Joint Conference on Natural Language Processing (ACL-IJCNLP) (Suntec, Singapore, 2009), pp. 221-224.

[100] Habash, N., and Sadat, F. Arabic Preprocessing Schemes for Statistical Machine Translation. In Proceedings of the Conference of the North American Chapter of the Association for Computational Linguistics (NAACL) (New York City, NY, 2006), pp. 49-52.

[101] Habash, N., Zalmout, N., Taji, D., Hoang, H., and Alzate, M. A parallel corpus for evaluating machine translation between Arabic and european languages. In Proceedings of the Conference of the European Chapter of the Association for Computational Linguistics (EACL) (Valencia, Spain, 2017), p. 235.

[102] HaBASH, N. Y. Introduction to Arabic natural language processing, vol. 3. Morgan \& Claypool Publishers, 2010.

[103] Hamdy Mubarak, Shimaa Amer, A. A., And Darwish, K. Arabic curriculum analysis. In The 28th International Conference on Computational Linguistics (COLING'2020) (2020).

[104] Hasan, S., El Isbihani, A., And Ney, H. Creating a large-scale Arabic to french statistical machinetranslation system. In LREC (2006), pp. 855858 .

[105] Hasanain, M., Barkallah, Y., Suwaileh, R., Kutlu, M., and ElSAYED, T. Artest: The first test collection for arabic web search with relevance rationales. In Proceedings of the 43rd International ACM SIGIR Conference on Research and Development in Information Retrieval (2020), pp. 2017-2020.

[106] Hasanain, M., Elsayed, T., and Magdy, W. Identification of answerseeking questions in arabic microblogs. In Proceedings of the 23rd ACM International Conference on Conference on Information and Knowledge Management (2014), pp. 1839-1842.

[107] Hasanain, M., Suwaileh, R., Elsayed, T., Kutlu, M., and AlmereKhi, H. Evetar: building a large-scale multi-task test collection over arabic tweets. Information Retrieval Journal 21, 4 (2018), 307-336.

[108] Hatem, A., And Nassar, A. Modified dijstra-like search algorithm for english to Arabic machine translation system. Proceedings EAMT 2008 (2008), 12th. 
[109] Hatem, A., And Omar, N. Syntactic reordering for Arabic-english phrase-based machine translation. In Database theory and application, bio-science and bio-technology. Springer, 2010, pp. 198-206.

[110] Hifny, Y., AND Ali, A. Efficient Arabic emotion recognition using deep neural networks. In ICASSP 2019-2019 IEEE International Conference on Acoustics, Speech and Signal Processing (ICASSP) (2019), IEEE, pp. $6710-6714$.

[111] Ibrahim-Sakre, M. M. A Fast and Expert Machine Translation System Involving Arabic Language. PhD thesis, Cranfield Institute of Technology, U.K., 1991.

[112] Inoue, G., Habash, N., Matsumoto, Y., and Aoyama, H. A parallel corpus of Arabic-Japanese news articles. In Proceedings of the Language Resources and Evaluation Conference (LREC) (Miyazaki, Japan, 2018).

[113] JaAfar, Y., AND BouzoubaA, K. A survey and comparative study of Arabic nlp architectures. In Intelligent Natural Language Processing: Trends and Applications. Springer, 2018, pp. 585-610.

[114] JARrAR, M. The Arabic ontology - an Arabic wordnet with ontologically clean content. Applied Ontology Journal (forthcoming).

[115] Jarrar, M., And Amayreh, H. An Arabic-multilingual database with a lexicographic search engine. In The 24th International Conference on Applications of Natural Language to Information Systems (NLDB 2019) (June 2019), vol. 11608 of LNCS, Springer, pp. 234-246.

[116] Jarrar, M., Amayreh, H., And McCrae, J. P. Representing Arabic lexicons in lemon - a preliminary study. In The 2nd Conference on Language, Data and Knowledge (LDK 2019) (May 2019), vol. 2402, CEUR, pp. 29-33.

[117] Jarrar, M., Habash, N., Alrimawi, F., Akra, D., and Zalmout, N. Curras: an annotated corpus for the Palestinian Arabic dialect. Language Resources and Evaluation (2016), 1-31.

[118] Khalifa, M., And ShaAlan, K. Character convolutions for Arabic named entity recognition with long short-term memory networks. Comput. Speech Lang. 58 (2019), 335-346.

[119] Khalifa, S., Habash, N., Abdulrahim, D., and Hassan, S. A Large Scale Corpus of Gulf Arabic. In Proceedings of the Language Resources and Evaluation Conference (LREC) (Portorož, Slovenia, 2016).

[120] Khalifa, S., Habash, N., Eryani, F., Obeid, O., Abdulrahim, D., AND KAABI, M. A. A morphologically annotated corpus of emirati Arabic. In Proceedings of the Language Resources and Evaluation Conference (LREC) (Miyazaki, Japan, 2018). 
[121] Khalifa, S., Hassan, S., And Habash, N. A Morphological Analyzer for Gulf Arabic Verbs. In Proceedings of the Workshop for Arabic Natural Language Processing (WANLP) (Valencia, Spain, 2017).

[122] Khalifa, S., Zalmout, N., And Habash, N. Yamama: Yet another multi-dialect Arabic morphological analyzer. In Proceedings of the International Conference on Computational Linguistics (COLING) (Osaka, Japan, 2016), pp. 223-227.

[123] Khalifa, S., Zalmout, N., And Habash, N. Morphological analysis and disambiguation for Gulf Arabic: The interplay between resources and methods. In Proceedings of the 12th Language Resources and Evaluation Conference (Marseille, France, May 2020), European Language Resources Association, pp. 3895-3904.

[124] Khalil, T., Halaby, A., Hammad, M. H., and El-Beltagy, S. R. Which configuration works best? An experimental study on Supervised Arabic Twitter Sentiment Analysis. In Proceedings of the First Conference on Arabic Computational Liguistics (ACLing 2015), co-located with CICLing 2015 (Cairo, Egypt, 2015), pp. 86-93.

[125] Lan, W., Chen, Y., Xu, W., and Ritter, A. Gigabert: Zero-shot transfer learning from english to Arabic, 2020.

[126] Liu, L., Shang, J., And Han, J. Arabic named entity recognition: What works and what's next. In Proceedings of the Fourth Arabic Natural Language Processing Workshop (Florence, Italy, Aug. 2019), Association for Computational Linguistics, pp. 60-67.

[127] Luqman, H., And Mahmoud, S. A. Automatic translation of Arabic text-to-Arabic sign language. Universal Access in the Information Society 18, 4 (2019), 939-951.

[128] Manmouri, M., Bies, A., Buckwalter, T., and Mekki, W. The Penn Arabic Treebank: Building a Large-Scale Annotated Arabic Corpus. In Proceedings of the International Conference on Arabic Language Resources and Tools (Cairo, Egypt, 2004), pp. 102-109.

[129] Maamouri, M., Bies, A., Kulick, S., Ciul, M., Habash, N., And EskAnDER, R. Developing an Egyptian Arabic Treebank: Impact of Dialectal Morphology on Annotation and Tool Development. In Proceedings of the Language Resources and Evaluation Conference (LREC) (Reykjavik, Iceland, 2014).

[130] Maamouri, M., Zaghouani, W., Cavalli-Sforza, V., Graff, D., AND Ciul, M. Developing aret: an nlp-based educational tool set for Arabic reading enhancement. In Proceedings of the Seventh Workshop on Building Educational Applications Using NLP (2012), pp. 127-135. 
[131] Magdy, W., Darwish, K., And El-Saban, M. Efficient languageindependent retrieval of printed documents without ocr. In International Symposium on String Processing and Information Retrieval (2009), Springer, pp. 334-343.

[132] Magdy, W., And Elsayed, T. Unsupervised adaptive microblog filtering for broad dynamic topics. Information Processing 83 Management 52, 4 (2016), 513-528.

[133] Mahmoud, M. H. A multiagents based intelligent tutoring system for teaching Arabic grammar. International Journal of Education and Learning Systems 3 (2018).

[134] Mahmoud, M. H., And El-Hamayed, S. H. A. An intelligent tutoring system for teaching the grammar of the Arabic language. Journal of Electrical Systems and Information Technology 3, 2 (2016), 282-294.

[135] Malhas, R., And Elsayed, T. Ayatec: Building a reusable verse-based test collection for arabic question answering on the holy qur'an. $A C M$ Transactions on Asian and Low-Resource Language Information Processing (TALLIP) 19, 6 (2020), 1-21.

[136] Meftouh, K., Harrat, S., Jamoussi, S., Abbas, M., and Smaili, K. Machine translation experiments on padic: A parallel Arabic dialect corpus. In Proceedings of the Pacific Asia Conference on Language, Information and Computation (2015).

[137] Mikolov, T., Corrado, G., Chen, K., and Dean, J. word2vec-v1. Proceedings of the International Conference on Learning Representations (ICLR 2013) (2013), 1-12.

[138] Mohammad, S. M., Bravo-Marquez, F., Salameh, M., and KirITChenko, S. Semeval-2018 Task 1: Affect in tweets. In Proceedings of International Workshop on Semantic Evaluation (SemEval-2018) (New Orleans, LA, USA, 2018).

[139] Mourad, A., And Darwish, K. Subjectivity and Sentiment Analysis of Modern Standard Arabic and Arabic Microblogs. Proceedings of the 4th Workshop on Computational Approaches to Subjectivity, Sentiment and Social Media Analysis, 3 (2013), 55-64.

[140] Mousser, J. A large coverage verb lexicon for Arabic. PhD thesis, University of Konstanz, Germany, 2013.

[141] Mubarak, H. Build fast and accurate lemmatization for arabic. In Proceedings of the Eleventh International Conference on Language Resources and Evaluation (LREC 2018) (2018). 
[142] Mubarak, H., Abdelali, A., Hassan, S., and Darwish, K. Spam detection on Arabic twitter. In International Conference on Social Informatics (2020), Springer, pp. 237-251.

[143] Mubarak, H., Abdelali, A., Sajjad, H., Samih, Y., and Darwish, K. Highly effective arabic diacritization using sequence to sequence modeling. In Proceedings of the 2019 Conference of the North American Chapter of the Association for Computational Linguistics: Human Language Technologies, Volume 1 (Long and Short Papers) (2019), pp. 2390-2395.

[144] Mubarak, H., and Darwish, K. Using Twitter to collect a multidialectal corpus of Arabic. In Proceedings of the Workshop for Arabic Natural Language Processing (WANLP) (Doha, Qatar, 2014).

[145] Mubarak, H., Darwish, K., And Magdy, W. Abusive language detection on Arabic social media. In Proceedings of the First Workshop on Abusive Language Online (2017), pp. 52-56.

[146] Mubarak, H., Darwish, K., Magdy, W., Elsayed, T., and AlKhAlifa, H. Overview of OSACT4 Arabic offensive language detection shared task. In Proceedings of the 4th Workshop on Open-Source Arabic Corpora and Processing Tools, with a Shared Task on Offensive Language Detection (Marseille, France, May 2020), European Language Resource Association, pp. 48-52.

[147] Nguyen, T., And Vogel, S. Context-based Arabic morphological analysis for machine translation. In CoNLL 2008: Proceedings of the Twelfth Conference on Computational Natural Language Learning (2008), pp. $135-142$.

[148] Nivre, J., Hall, J., And Nilsson, J. MaltParser: A data-driven parser-generator for dependency parsing. In Proceedings of the Language Resources and Evaluation Conference (LREC) (Genoa, Italy, 2006), pp. 2216-2219.

[149] OARD, D. W., AND Gey, F. C. The trec 2002 arabic/english clir track. In TREC (2002).

[150] Obeid, O., Salameh, M., Bouamor, H., and Habash, N. ADIDA: Automatic dialect identification for Arabic. In Proceedings of the 2019 Conference of the North American Chapter of the Association for Computational Linguistics (Demonstrations) (Minneapolis, Minnesota, June 2019).

[151] Obeid, O., Zalmout, N., Khalifa, S., Taji, D., Oudah, M., Alhafni, B., Inoue, G., Eryani, F., Erdmann, A., and Habash, N. CAMeL tools: An open source python toolkit for Arabic natural language processing. In Proceedings of the 12th Language Resources and Evaluation Conference (Marseille, France, May 2020), European Language Resources Association, pp. 7022-7032. 
[152] Olive, J., Christianson, C., And McCary, J. Handbook of natural language processing and machine translation: DARPA global autonomous language exploitation. Springer Science \& Business Media, Verlag New York, 2011.

[153] Olive, J., Christianson, C., And McCary, J. Handbook of natural language processing and machine translation: DARPA global autonomous language exploitation. Springer Science \& Business Media, 2011.

[154] Oudah, M., Almahairi, A., and Habash, N. The impact of preprocessing on Arabic-English statistical and neural machine translation. In Proceedings of Machine Translation Summit XVII Volume 1: Research Track (Dublin, Ireland, Aug. 2019), European Association for Machine Translation, pp. 214-221.

[155] Parker, R., Graff, D., Chen, K., Kong, J., and Maeda, K. Arabic Gigaword Fifth Edition. LDC catalog number No. LDC2011T11, ISBN 1-58563-595-2, 2011.

[156] Pasha, A., Al-Badrashiny, M., Diab, M. T., El Kholy, A., Eskander, R., Habash, N., Pooleery, M., Rambow, O., and Roth, R. Madamira: A fast, comprehensive tool for morphological analysis and disambiguation of Arabic. In Lrec (2014), vol. 14, pp. 1094-1101.

[157] Pennington, J., Socher, R., And Manning, C. D. Glove: Global vectors for word representation. In Proceedings of the 2014 conference on empirical methods in natural language processing (EMNLP) (2014), pp. 1532-1543.

[158] Rafalovitch, A., Dale, R., et Al. United nations general assembly resolutions: A six-language parallel corpus. In Proceedings of the $M a$ chine Translation Summit (MT Summit) (Ottawa, Canada, 2009), vol. 12, pp. 292-299.

[159] Rafea, A., Sabry, M., El-Ansary, R., and Samir, S. Al-mutargem: a machine translator for middle east news. In Proceedings of the 3rd International Conference and Exhibition on Multi-lingual Computing (1992).

[160] Refaee, E., and Rieser, V. Subjectivity and Sentiment Analysis of Arabic Twitter Feeds with Limited Resources. In Proceedings of the Workshop on Free/Open-Source Arabic Corpora and Corpora Processing Tools (Reykjavik, Iceland, 2014), pp. 16-21.

[161] Romeo, S., Da San Martino, G., Belinkov, Y., Barrón-Cedeño, A., Eldesouki, M., Darwish, K., Mubarak, H., Glass, J., And Moschitti, A. Language processing and learning models for community question answering in Arabic. Information Processing $\mathcal{G}$ Management 56, 2 (2019), 274-290. 
[162] Rosenthal, S., Farra, N., And Nakov, P. SemEval-2017 task 4: Sentiment analysis in Twitter. In Proceedings of the 11th International Workshop on Semantic Evaluation (Vancouver, Canada, August 2017), SemEval '17, Association for Computational Linguistics.

[163] Rytting, C. A., Rodrigues, P., Buckwalter, T., Novak, V., Bills, A., Silbert, N. H., And Madgavkar, M. ArCADE: An Arabic corpus of auditory dictation errors. In Proceedings of the Ninth Workshop on Innovative Use of NLP for Building Educational Applications (Baltimore, Maryland, June 2014), Association for Computational Linguistics, pp. 109-115.

[164] Sadat, F., Kazemi, F., And Farzindar, A. Automatic Identification of Arabic Language Varieties and Dialects in Social Media. In Proceedings of the Second Workshop on Natural Language Processing for Social Media (SocialNLP) (Dublin, Ireland, 2014).

[165] Saddiki, H., Habash, N., Cavalli-Sforza, V., and Al Khalil, M. Feature optimization for predicting readability of Arabic L1 and L2. In Proceedings of the 5th Workshop on Natural Language Processing Techniques for Educational Applications (Melbourne, Australia, July 2018), Association for Computational Linguistics, pp. 20-29.

[166] Safaya, A., Abdullatif, M., and Yuret, D. Kuisail at semeval-2020 task 12: Bert-cnn for offensive speech identification in social media, 2020.

[167] Sajuad, H., Darwish, K., And Belinkov, Y. Translating dialectal Arabic to English. In Proceedings of the Conference of the Association for Computational Linguistics (ACL) (Sofia, Bulgaria, 2013), pp. 1-6.

[168] Sajuad, H., Durrani, N., Guzman, F., Nakov, P., Abdelali, A., Vogel, S., Salloum, W., Kholy, A. E., and Habash, N. Egyptian Arabic to english statistical machine translation system for nist openmt'2015. arXiv preprint arXiv:1606.05759 (2016).

[169] Salama, A., Bouamor, H., Mohit, B., and Oflazer, K. YouDACC: the Youtube Dialectal Arabic Comment Corpus. In Proceedings of the Language Resources and Evaluation Conference (LREC) (Reykjavik, Iceland, 2014), pp. 1246-1251.

[170] Salameh, M., Bouamor, H., And Habash, N. Fine-grained Arabic dialect identification. In Proceedings of the International Conference on Computational Linguistics (COLING) (Santa Fe, New Mexico, USA, 2018), pp. 1332-1344.

[171] Salameh, M., Mohammad, S., and Kiritchenko, S. Sentiment after Translation: A Case-Study on Arabic Social Media Posts. In Proceedings of the 2015 Conference of the North American Chapter of the Association for Computational Linguistics: Human Language Technologies (Denver, Colorado, 2015), Association for Computational Linguistics, pp. 767-777. 
[172] Salloum, W., Elfardy, H., Alamir-Salloum, L., Habash, N., And DiAB, M. Sentence Level Dialect Identification for Machine Translation System Selection. In Proceedings of the 52nd Annual Meeting of the Association for Computational Linguistics (Baltimore, Maryland, USA, 2014).

[173] Salloum, W., and Habash, N. Dialectal to Standard Arabic Paraphrasing to Improve Arabic-English Statistical Machine Translation. In Proceedings of the First Workshop on Algorithms and Resources for Modelling of Dialects and Language Varieties (Edinburgh, Scotland, 2011), pp. 10-21.

[174] Samin, Y., Attia, M., Eldesouki, M., Abdelali, A., Mubarak, H., KAllmeyer, L., AND DARWish, K. A neural architecture for dialectal Arabic segmentation. In Proceedings of the Workshop for Arabic Natural Language Processing (WANLP) (Valencia, Spain, 2017), pp. 46-54.

[175] Sawaf, H. Arabic dialect handling in hybrid machine translation. In Proceedings of the Conference of the Association for Machine Translation in the Americas (AMTA) (Denver, Colorado, 2010).

[176] Sghaier, M. A., And ZRigui, M. Tunisian dialect-modern standard Arabic bilingual lexicon. In 2017 IEEE/ACS 14th International Conference on Computer Systems and Applications (AICCSA) (2017), IEEE, pp. 973-979.

[177] Shaalan, K. A survey of Arabic named entity recognition and classification. Computational Linguistics 40 (2014), 469 - 510.

[178] Shaalan, K., Bakr, H., and Ziedan, I. Transferring Egyptian colloquial dialect into modern standard Arabic. In Proceedings of the Conference on Recent Advances in Natural Language Processing (RANLP) (2007), pp. 525-529.

[179] Shadlan, K., Rafea, A., Moneim, A. A., and Baraka, H. Machine translation of english noun phrases into Arabic. International Journal of Computer Processing of Oriental Languages 17, 02 (2004), 121-134.

[180] Shanlan, K., And Talhami, H. Error analysis and handling in Arabic icall systems. In Artificial Intelligence and Applications (2006), Citeseer, pp. 109-114.

[181] ShaAlan, K. F. An intelligent computer assisted language learning system for Arabic learners. Computer Assisted Language Learning 18, 1-2 (2005), 81-109.

[182] Shahin, I., Nassif, A. B., And Hamsa, S. Emotion recognition using hybrid gaussian mixture model and deep neural network. IEEE Access 7 (2019), 26777-26787. 
[183] Shahrour, A., Khalifa, S., Taji, D., and Habash, N. CamelParser: A system for Arabic syntactic analysis and morphological disambiguation. In Proceedings of the International Conference on Computational Linguistics (COLING) (2016), pp. 228-232.

[184] Shawar, A., And Atwell, E. An Arabic chatbot giving answers from the qur'an. In Proceedings of TALN04: XI Conference sur le Traitement Automatique des Langues Naturelles (2004), vol. 2, ATALA, pp. 197-202.

[185] Shoukry, A., And Rafea, A. A Hybrid Approach for Sentiment Classification of Egyptian Dialect Tweets. In First International Conference on Arabic Computational Linguistics (ACLing) (Cairo, Egypt, 2015), pp. 7885.

[186] Smä̈li, K., Abbas, M., Meftouh, K., and Harrat, S. Building resources for Algerian Arabic dialects. In Proceedings of the Conference of the International Speech Communication Association (Interspeech) (2014).

[187] Smrž, O. ElixirFM - Implementation of Functional Arabic Morphology. In Proceedings of the Workshop on Computational Approaches to Semitic Languages (CASL) (Prague, Czech Republic, 2007), ACL, pp. 1-8.

[188] Smrž, O., Šnaidauf, J., And Zemánek, P. Prague Dependency Treebank for Arabic: Multi-Level Annotation of Arabic Corpus. In Proceedings of the International Symposium on Processing of Arabic (Manouba, Tunisia, 2002), pp. 147-155.

[189] Soliman, A. B., Eissa, K., and El-Beltagy, S. R. AraVec: A set of Arabic Word Embedding Models for use in Arabic NLP. In Procedia Computer Science (2017).

[190] Suwaileh, R., Kutlu, M., Fathima, N., Elsayed, T., and Lease, M. Arabicweb16: A new crawl for today's arabic web. In Proceedings of the 39th International ACM SIGIR conference on Research and Development in Information Retrieval (2016), pp. 673-676.

[191] Tachicart, R., BouzoubaA, K., And JaAfar, H. Building a moroccan dialect electronic dictionary (mded). In 5th International Conference on Arabic Language Processing (2014), pp. 216-221.

[192] Tahir, G. R., Asghar, S., And Masood, N. Knowledge based machine translation. In 2010 International Conference on Information and Emerging Technologies (2010), IEEE, pp. 1-5.

[193] Taji, D., El Gizuli, J., and Habash, N. An Arabic dependency treebank in the travel domain. In Proceedings of the Workshop on OpenSource Arabic Corpora and Processing Tools (OSACT) (Miyazaki, Japan, 2018). 
[194] TAJi, D., Habash, N., AND Zeman, D. Universal dependencies for Arabic. In Proceedings of the Workshop for Arabic Natural Language Processing (WANLP) (Valencia, Spain, 2017).

[195] Taji, D., Khalifa, S., Obeid, O., Eryani, F., and Habash, N. An Arabic Morphological Analyzer and Generator with Copious Features. In Proceedings of the Fifteenth Workshop on Computational Research in Phonetics, Phonology, and Morphology (SIGMORPHON) (2018), pp. 140150 .

[196] Tiedemann, J. Parallel corpora for everyone. Baltic J. Modern Comput.(BJMC) 4, 2 (2016).

[197] Versteegh, K. Arabic language. Edinburgh University Press, 2014.

[198] Zaghounin, W. Critical Survey of the Freely Available Arabic Corpora. In Proceedings of the Workshop on Open-Source Arabic Corpora and Processing Tools (OSACT) (2014), pp. 1-8.

[199] Zaghounini, W., And Charfi, A. ArapTweet: A Large Multi-Dialect Twitter Corpus for Gender, Age and Language Variety Identification. In Proceedings of the Language Resources and Evaluation Conference (LREC) (Miyazaki, Japan, 2018).

[200] Zaghouani, W., Habash, N., Bouamor, H., Rozovskaya, A., MoHit, B., Heider, A., AND Oflazer, K. Correction annotation for non-native Arabic texts: Guidelines and corpus. In Proceedings of the Linguistic Annotation Workshop (LAW) (2015), pp. 129-139.

[201] Zaghouani, W., Mohit, B., Habash, N., Obeid, O., Tomeh, N., Rozovskaya, A., Farra, N., Alkuhlani, S., and Oflazer, K. Large Scale Arabic Error Annotation: Guidelines and Framework. In Proceedings of the Language Resources and Evaluation Conference (LREC) (Reykjavik, Iceland, 2014).

[202] Zaidan, O., And Callison-Burch, C. Arabic dialect identification. Computational Linguistics (2013).

[203] Zaidan, O. F., And Callison-Burch, C. The Arabic online commentary dataset: an annotated dataset of informal Arabic with high dialectal content. In Proceedings of the 49th Annual Meeting of the Association for Computational Linguistics: Human Language Technologies: short papers-Volume 2, Organization = Association for Computational Linguistics (2011), pp. 37-41.

[204] Zalmout, N., Erdmann, A., And Habash, N. Noise-robust morphological disambiguation for dialectal Arabic. In Proceedings of the Conference of the North American Chapter of the Association for Computational Linguistics (NAACL) (New Orleans, Louisiana, USA, 2018). 
[205] Zalmout, N., And Habash, N. Don't throw those morphological analyzers away just yet: Neural morphological disambiguation for Arabic. In Proceedings of the Conference on Empirical Methods in Natural Language Processing (EMNLP) (Copenhagen, Denmark, 2017), pp. 704-713.

[206] Zalmout, N., And Habash, N. Optimizing Tokenization Choice for Machine Translation across Multiple Target Languages. The Prague Bulletin of Mathematical Linguistics 108 (2017), 257-270.

[207] Zampieri, M., Malmasi, S., Nakov, P., Ali, A., Shon, S., Glass, J., Scherrer, Y., Samardžić, T., Luubešić, N., Tiedemann, J., van der Lee, C., Grondelaers, S., Oostdijk, N., Speelman, D., van den Bosch, A., Kumar, R., Lahiri, B., And Jain, M. Language identification and morphosyntactic tagging: The second VarDial evaluation campaign. In Proceedings of the Fifth Workshop on NLP for Similar Languages, Varieties and Dialects (VarDial 2018) (Santa Fe, New Mexico, USA, Aug. 2018), Association for Computational Linguistics, pp. 1-17.

[208] Zbib, R., Malchiodi, E., Devlin, J., Stallard, D., Matsoukas, S., Schwartz, R., Makhoul, J., Zaidan, O. F., and Callison-Burch, C. Machine Translation of Arabic Dialects. In Proceedings of the Conference of the North American Chapter of the Association for Computational Linguistics (NAACL) (Montréal, Canada, 2012), pp. 49-59.

[209] Zeroual, I., and Lakhouaja, A. A new quranic corpus rich in morphosyntactical information. International Journal of Speech Technology 19, 2 (2016), 339-346.

[210] Zerrouki, T., And Balla, A. Tashkeela: Novel corpus of Arabic vocalized texts, data for auto-diacritization systems. Data in brief 11 (2017), 147 .

[211] Zhang, Y., Li, C., Barzilay, R., and Darwish, K. Randomized greedy inference for joint segmentation, pos tagging and dependency parsing. In Proceedings of the 2015 Conference of the North American Chapter of the Association for Computational Linguistics: Human Language Technologies (2015), pp. 42-52.

[212] Zhu, M., Zhang, Y., Chen, W., Zhang, M., and Zhu, J. Fast and accurate shift-reduce constituent parsing. In Proceedings of the 51st Annual Meeting of the Association for Computational Linguistics (Volume 1: Long Papers) (2013), pp. 434-443. 